\title{
Electrophysiological and firing properties of neurons: categorizing soloists and choristers in primary visual cortex
}

Lyes Bachatene ${ }^{a, b}$, Vishal Bharmauria ${ }^{a, b}$, Sarah Cattan ${ }^{a, b}$, Nayan Chanauria ${ }^{a, b}$, Jean Rouat ${ }^{\mathrm{a}, \mathrm{b}}$ and Stéphane Molotchnikoff ${ }^{\mathrm{a}, \mathrm{b}, *}$

'Laboratoire de Neurosciences de la vision, Département de Sciences Biologiques, Université de Montréal, Montréal, QC, Canada

${ }^{\text {b}}$ Neurosciences Computationnelles et Traitement Intelligent des Signaux (NECOTIS, Université de Sherbrooke, Québec, Canada)

${ }^{*}$ Correspondence to: Stéphane Molotchnikoff, Ph.D

Département de Sciences Biologiques

Université de Montréal

CP 6128 Succ. Centre-Ville

Montréal, QC, Canada, H3C 3J7

Tel : +15143436616

e-mail : stephane.molotchnikoff@umontreal.ca

\section{ABSTRACT}

Visual processing in the cortex involves various aspects of neuronal properties such as morphological, electrophysiological and molecular. In particular, the neural firing pattern is an important indicator of dynamic circuitry within a neuronal population. Indeed, in microcircuits, neurons act as soloists or choristers wherein the characteristical activity of a 'soloist' differs from the firing pattern of a 'chorister'. Both cell types correlate their respective firing rate with the global populational activity in a unique way. In the present study, we sought to examine the relationship between the spike shape (thin spike neurons and broad spike neurons) of cortical neurons recorded from V1, their firing levels and 
their propensity to act as soloists or choristers. We found that thin spike neurons, which exhibited higher levels of firing, generally correlate their activity with the neuronal population (choristers). On the other hand, broad spike neurons showed lower levels of firing and demonstrated weak correlations with the assembly (soloists). A major consequence of the present study is: estimating the correlation of neural spike trains with their neighboring population is a predictive indicator of spike waveforms and firing level. Indeed, we found a continuum distribution of coupling strength ranging from weak correlation-strength (attributed to low-firing neurons) to high correlation-strength (attributed to highfiring neurons). The tendency to exhibit high- or low-firing is conducive to the spike shape of neurons. Our results offer new insights into visual processing by showing how high-firing rate neurons (mostly thin spike neurons) could modulate the neuronal responses within cell-assemblies.

Keywords: soloists, choristers, visual cortex, thin spike neurons, broad spike neurons, visual processing, firing rate.

\section{Abbreviations:}

CCG: cross-correlogram

HF: high firing

LF: low firing

\section{Introduction}


Sensory information is represented in the cortex by networks of co-activated neurons which coordinate their firing activity, thus forming functional assemblies [25]. Encoding stimulus attributes involves a variety of strategies considering the anatomical and functional divergence within neuronal populations. Indeed, based on their intrinsic electrophysiological properties, cortical cells are classified into different types such as regular-spiking (RS), fast-rhythmic-bursting (FRB), fastspiking (FS) and intrinsically bursting (IB) neurons [35]. Despite the fact that neurons exhibit large panoply of firing properties, their correlated activities within assemblies efficiently code the stimulus features rather than the independent spiking of each involved neuron [25]. As a matter of fact, it is largely reported that neurons coding for similar attributes exhibit high levels of correlation and are thus functionally strongly connected to each other $[1,7,12,20,34,39]$ yet, the debate remains controversial [28, 29, 32, 38]. However, in spite of the stimulus-selective correlation aspect, each neuron may also show coupling with its neighboring local population [27] as if it belongs to a "neuronal orchestra" [15]. It was recently shown in the mouse primary visual cortex and monkey area V4 that, regardless of neuronal preferences, population coupling may lead to discrimination between the choristers (highly coupled) and soloists (weakly coupled) [27]. These two groups differ in many aspects. For instance, highly correlated neurons may receive stronger synaptic drive from neighboring cells [27]. In addition, noise correlation $\left(R_{s c}\right)$ is higher for choristers than soloists in IT cortex [18]. Anatomical differences were also observed since the cortical laminar distribution for these types of units is distinct [18]. In mouse V1, population coupling varies between 
the narrow-spiking and regular-spiking neurons; however, this difference was reported to be smaller than the class-variability [27]. In the present investigation, we sought to examine the relationship between the cell-type (thin spike and broad spike neurons), the firing rate (high-firing and low-firing) and the population coupling of above classes in adult anaesthetized cats (columnar-organized cortex). We found that thin spike neurons systematically exhibited higher firing levels than broad spike neurons. In addition, high-firing (HF) neurons displayed higher coupling strength than the low-firing (LF) neurons.

We report that within a sub-network, LF neurons (which were mostly broad spike neurons and weakly coupled) and HF neurons (mainly thin spike neurons and highly coupled) likely play distinct roles in information coding where high-firing neurons may orchestrate the sensory selectivity of the neighboring cells.

\section{Materials and methods}

\subsection{Ethical Approval}

Animal surgery procedures and electrophysiological recordings followed the guidelines of the Canadian Council on Animal Care and were approved by the Institutional Animal Care and Use Committee of the University of Montreal. Animals were supplied by the Division of Animal Resources of the University of Montreal. The experiments were conducted in accordance with the Guide for Care and Use of Laboratory Animals of the National Institutes of Health (USA). 
Details of animal surgery, electrophysiology, visual stimulation and isolation of neurons are described in Bachatene et al., 2015 [4].

\subsection{Cross-correlation computations}

Cross-correlogram shows the conditional probability of a spike at time $t_{0}+t$ on the condition that there is a reference event (or reference spike, spike-by-spike analysis) at time $t_{0}$. The spike train of each neuron was cross-correlated with the population rate activity (the multi-unit activity) at its preferred orientation (Fig. 1A). Time axis is divided into bins. The first bin is defined as: XMin, XMin+Bin. The next bin is XMin+Bin, XMin+Bin*2, etc. We calculated the distances from each spike to all spikes of the spike train as follows:

$d[i]=t s[i]-\operatorname{ref}[k]$

where ts[i] represents the spike train, and ref[k] is each timestamp.

Bin counts were then divided by the number of reference events to normalize the counts per bin into probabilities. 95\% statistical threshold for the significance of the bins was used. Each bin-width was set at $1 \mathrm{~ms}$ and a Gaussian filter width of 3 bins was used. Probabilities were calculated from the counts/bin as follows:

$$
P=\frac{N}{T} \times b
$$


where $N / T$ is the neuron frequency and $b$ represents the bin size of the calculated firing of the neuron. $T$ represents the total time interval and $\mathrm{N}$ the number of spikes within this interval.

The 95\% confidence limit was calculated assuming that the expected bin count (C) has a Poisson distribution:

$C=P \times N r e f$

where Nref is the number of reference events.

The $95 \%$ confidence limit is calculated as follows:

Low Conf.$=x$ such that Prob $(S<x)=0.005$

High Conf.$=y$ such that Prob $(S>y)=0.005$

where S represents a random variable which has a Poisson distribution with parameter $\mathrm{C}$.

Raw cross-correlograms were corrected by using a shift-predictor procedure in order to eliminate the putative significant peaks resulting from the coincident firing due to simultaneous visual drive during each trial $[4,13]$.

\section{Results}

\subsection{Coupling strength strategy}

We performed electrophysiological recordings in primary visual cortex of adult anaesthetized cats $(n=8)$ that were visually stimulated (drifting sine-wave 
grating patches covering the excitatory fields, spatial frequency $=0.24 \mathrm{cycle} /{ }^{\circ}$, temporal frequency $=1.0-2.0 \mathrm{~Hz}$, contrast $=80 \%$, orientations: from $0^{\circ}$ to $157.5^{\circ}$, 25 trials, stimulus duration: 4.1s [5]). Recordings were performed from multiple cortical sites using multi-channel electrodes. Spiking responses were sorted from the multi-unit activity. In addition to the distinct waveforms, the isolation of cells was based on the classical procedure, i.e., principal component analysis, autocorrelograms and cluster separation (Mahalanobis distance and mean cluster distance) $[5,8]$.

We cross-correlated the firing activity of each neuron with the firing of the global population of neurons (multi-unit activity) using the shift-corrected crosscorrelation computation (see materials and methods for details). Figure $1 \mathrm{~A}$ shows the population coupling strategy: raster plots of three simultaneously recorded cells and their coupling strength indicated by the peak straddling zero in the corresponding correlograms along with the population rate (three superimposed cross-correlograms with the respective color code for each neuron) are displayed. In this example, the green cell exhibited larger correlation-strength than the blue and purple cells. The histogram distribution of all values for correlation-strengths is displayed in Fig. 1B. Note that the values of coupling strengths were normalized (minimum value corresponds to zero, maximum value corresponds to 1 ) due to the variability between all experiments ( $n=73$ neurons). The absence of a bimodal mode suggests that the coupling strength ranges from low to high values. However, differences were observed based on two parameters: the spike-type and the firing rate (see next sections). 


\subsection{Spike-types and coupling strength}

In total, 73 neurons were isolated and classified into two major types, namely thin spike neurons $(n=30)$ and broad spike neurons $(n=43)$. Spike-width was quantified as the interval between the trough and peak of the average spike wave-shape of the neuron, allowing for the distinction between broad waveforms (spike-width $\geq 0.5 \mathrm{~ms}$ ) and narrow waveforms (spike-width $<0.5 \mathrm{~ms}$ ) $[4,9,22$, 31]. An example is shown in Fig. $2 A$ where thin spike neurons and broad spike cells have distinct waveform with a trough-to-peak latency of $0.33 \mathrm{~ms}$ and 0.55 ms, respectively. Superimposed spike-waveforms of 20 cells are shown for each type in Fig. 2B. The bold traces represent the average waveforms. Figure $2 \mathrm{C}$ illustrates the distribution of the thin spike neurons (black) and the broad spike neurons (orange) depending on their trough-to-peak interval (trough-to-peak interval $<0.5 \mathrm{~ms}$, mean $=0.30 \pm 0.04 \mathrm{~ms}$, for thin spike cells, trough-to-peak interval $\geq 0.5 \mathrm{~ms}$, mean $=0.52 \pm 0.04 \mathrm{~ms}$, for broad spike cells $[4,9,22,31])$ Averaged spike-widths are displayed in Fig. 2D.

Figure $2 \mathrm{E}$ illustrates the respective cross-correlation of a thin spike neuron and a broad spike neuron (the spike shapes are represented above each CCG) with the populational activity. Indeed, the correlation-strength indicated by the significant peaks in the cross-correlograms was notably different for the thin spike neurons (black CCG, CCG magnitude $=0.005$ ) and the broad spike neurons (orange CCG, CCG magnitude $=0.0003$ ). Figures $2 \mathrm{~F}$ and $\mathrm{G}$ display examples of cross-correlograms between the populational activity and the firing 
rate of thin spike cells (black) and broad spike cells (orange), respectively. This tendency was observed for all sampled neurons. The averaged values were $0.003 \pm 0.0009$ and $0.001 \pm 0.0004$ for thin spike cells and broad spike cells, respectively ( $t$-test, $\mathrm{p}<0.05$, Fig. $2 \mathrm{H}$ ).

\subsection{Firing rate, spike-type and categorization of soloists and choristers}

Correlating the spike trains of each neuron with the population rate generates two notable behaviors within the assembly: neurons acting as soloists show a weak correlation index whereas neurons acting as choristers exhibit strong correlation with the population activity. It has been suggested that soloists are distinct from choristers in many aspects such as the synaptic connectivity [27] and the laminar distribution [18]. We examined the relationship between the waveform type, the firing rate and the correlation-strength of the cells' firing with the population activity. Typical examples of response-frequency plots to optimal orientation are shown in Fig. 3A (same neurons as in Fig. 2F, G). The relationship between the firing rate and the presented oriented stimuli is displayed in Fig. 3B; polar plots of two neurons (thin and broad spikes) exhibit differences in their orientation tuning and firing rate. It may be worthwhile to underline that poorly tuned cells are often associated with thin spike neurons whereas highly tuned neurons are related to broad spike neurons $[2,3,6,10,16$, $17,21,24,36]$. 
Moreover, we found that thin spike cells presented higher levels of firing rate in comparison to broad spike cells (responses measured for optimal orientations, Figure $3 \mathrm{C}$, $t$-test, $\mathrm{p}<0.01)$.

In addition, we sought to examine the relationship between the firing rate and the coupling strength. Figure 3D illustrates these correlations for all experiments. We observed significantly higher correlations between the firing rate and the coupling activity as the R-squared ranged from 0.75 to 0.99 .

\section{Discussion}

In the current study, we classified simultaneously recorded neurons from primary visual cortex of adult anaesthetized cats into thin spike neurons and broad spike neurons and studied their firing rate patterns. These two parameters (spike waveforms and firing rate level) were then related to the coupling strength of neurons with the neighboring population. The firing activity of a neuron characterized as a 'chorister' is highly correlated with the firing rate of the global population. On the other hand, a 'soloist' tends to be more autonomous, exhibiting independent firing $[23,27]$. Here we found a systematic relationship between the cell-class, the firing pattern and the correlation-strength of neurons. Despite the large diversity of cell-types, cortical neurons coordinate their spiking activity forming functional sub-networks which, when co-activated encode the attributes of stimuli [25]. It is extensively reported that sensory neurons may exhibit different levels of correlation depending largely on their selectivity to 
specific sets of stimuli $[1,7,12,20,34,39]$, though the debate persists $[28,29$, 32, 38].

It has been reported that the correlation properties are inherent characteristics of neurons [27]. Indeed, for both stimulation condition and spontaneous activity, soloists and choristers maintain their distinct firing-patterns such as carrying more general information for choristers than soloists $[23,27]$. On other hand, this correlation seems to be independent of neuronal sparseness and plays a key role in predicting visual search efficiency in humans [18].

Moreover, in rodent sensory cortex, it has been shown that soloists and choristers can be dissociated based on several features. Soloists were reported to be less functionally connected as they were driven less effectively by optogenetic activation [27]. Significant distinction in noise correlation, which represents the correlation coefficient of spike count responses to the presentation of a stimulus [11], has also been reported; noise correlation $\left(R_{s c}\right)$ was much higher for choristers than for soloists in IT cortex [18]. Laminar differences were observed in macaque IT where soloists are common at 0.2 and $1.2 \mathrm{~mm}$ depth, whereas choristers are rare in layer 4 and are located in nongranular output layers [18]. In the present investigation, we report that in cat's primary visual cortex, which exhibits a columnar organization, thin spike neurons exhibited higher firing levels and tend to differ in their coupling with their ensemble from broad spike neurons exhibiting lower firing levels and weaker population coupling. It has been reported that thin spike neurons and broad spike neurons exhibit different tuning properties $[2,3,6,10,16,17,21,24,36]$; thin 
spike cells being poorly tuned to orientation stimuli whereas broad spike cells being more highly tuned.

Furthermore, in cat's visual cortex, large number of excitatory neurons exhibit narrow spikes making the spike-width a non-valid parameter, therefore not permitting the distinction between pyramidal cells and inhibitory interneurons [26]. A recent study demonstrated that in cat $\mathrm{V} 1$, neurons show similar diversity in their spike-widths. In addition, neurons with a broad spike exhibit higher correlation levels between each other [31]. On the contrary, we found that correlating the spiking activity of each spike-waveform with the population activity shows the opposite trend: cells with a narrow spike-shape (also highly firing) exhibited greater coupling strengths than cells with a broad spike-shape. It is to be emphasized that on a cell-pair basis, the firing rates and peak magnitudes in CCG's are unrelated [14, 30, 33].

Complex circuitry mechanisms of individual neurons and the assemblies they form are involved in visual information processing [19]. Thus, measuring correlated spike-trains between neurons and the entire group predicts the spike shape and the firing rate pattern as the coupling level can be distinguishable between broad spike neurons (mainly LF neurons) and thin spike neurons (mainly HF neurons). Synchrony within a restricted space such as a cortical orientation domain can be accomplished by their specific relationships [37]. Combined with our recent finding which confers to the thin spike neurons higher gamma activity [8], these findings provide new insights into visual information processing. Indeed, within a cell-assembly comprising different cell-types, HF 
neurons may modulate the inputs and contribute to the selectivity of broad spike neurons in a recurrent "broad spike - thin spike" neuronal ensemble. A

schematic model of a neuronal population is illustrated in Fig. 4 where a stimulus is coded by a cell-assembly; the HF neurons (black) are more correlated with the neuronal ensemble. This may occur to ensure an efficient firing of neurons preferring the presented stimulus. LF neurons (orange) are less coupled to the population but exhibit correlations with neighboring neurons preferring the same orientation.

\section{Disclosure statement}

The authors have no conflicts of interest to declare.

\section{Legends to figures}

Fig. 1. Correlation strategy.

(A) Schematic representation of the correlation procedure between three recorded neurons and the populational rate. Raster plots of the spiking activity of the neurons and respective superimposed cross-correlograms are displayed. (B) Histogram distribution of the coupling strengths for all experiments $(n=73)$.

Fig. 2. Population coupling and types of neurons.

(A) Examples of two simultaneously recoded neurons showing the measurements of the spike-widths (trough-to-peak intervals). (B) Superimposed spike-waveforms of 20 cells from each type. The bold traces represent the average waveforms. (C) Histogram distribution of the trough-to-peak intervals for all neurons $(n=73)$. Orange and black histograms reflect the broad spike and the thin spike cells, respectively. (D) Averaged spike-widths for each cell-type. (E) Cross-correlograms between two neurons (the respective spike-waveforms are 
shown above each CCG) and the global spiking activity of the neuronal population. $X$-axis represents the time-window and $Y$-axis indicates the magnitude of the CCG. ( $F$ and $G$ ) Cross-correlograms between a group of thin spike cells (black) and the population rate $(F)$, and between broad spike cells (orange) and the populational rate $(\mathrm{G})$. $(\mathrm{H})$ Global statistics on the correlationstrength for all neurons $(n=73)$.

Fig. 3. Firing rate, spike-type and coupling strength.

(A) Frequency plots of typical examples of thin spike neurons (black) and broad spike neurons (orange). Note the differences on Y-axis scales. (B) Polar plots of two neurons showing the relationship between the stimulus and the firing rate. The broad spike neuron (orange) exhibit higher selectivity (sharply tuned). (C) Global statistics on the difference in firing rate levels between thin spike neurons and broad spike neurons. (D) Regression analyses between the firing rate and the correlation-strength for all experiments. R-squared values are indicated for each graphic.

Fig. 4. Schematic model of a neuronal ensemble and the respective putative functional connections between thin spike neurons (HF neurons) and broad spike cells (LF neurons).

\section{Acknowledgements}

Funding were supported by CRSNG (Conseil de Recherches en Sciences Naturelles et en Génie) and FRQ-NT (Fonds de recherche du Québec - Nature et technologies).

\section{References}

[1] K.D. Alloway, S.A. Roy, Conditional cross-correlation analysis of thalamocortical neurotransmission, Behavioural brain research 135 (2002) 191-196.

[2] B.V. Atallah, W. Bruns, M. Carandini, M. Scanziani, Parvalbumin-expressing interneurons linearly transform cortical responses to visual stimuli, Neuron 73 (2012) 159-170. 
[3] R. Azouz, C.M. Gray, L.G. Nowak, D.A. McCormick, Physiological properties of inhibitory interneurons in cat striate cortex, Cerebral cortex 7 (1997) 534-545.

[4] L. Bachatene, V. Bharmauria, S. Cattan, J. Rouat, S. Molotchnikoff, Modulation of functional connectivity following visual adaptation: homeostasis in V1, Brain research 1594 (2015) 136-153.

[5] L. Bachatene, V. Bharmauria, S. Cattan, J. Rouat, S. Molotchnikoff, Reprogramming of orientation columns in visual cortex: a domino effect, Scientific reports 5 (2015) 9436.

[6] L. Bachatene, V. Bharmauria, J. Rouat, S. Molotchnikoff, Adaptation-induced plasticity and spike waveforms in cat visual cortex, Neuroreport 23 (2012) 88-92.

[7] P. Bartho, H. Hirase, L. Monconduit, M. Zugaro, K.D. Harris, G. Buzsaki, Characterization of neocortical principal cells and interneurons by network interactions and extracellular features, Journal of neurophysiology 92 (2004) 600-608.

[8] V. Bharmauria, L. Bachatene, S. Cattan, N. Chanauria, J. Rouat, S. Molotchnikoff, Stimulus-dependent augmented gamma oscillatory activity between the functionally connected cortical neurons in the primary visual cortex, The European journal of neuroscience (2015).

[9] D.S. Bortone, S.R. Olsen, M. Scanziani, Translaminar inhibitory cells recruited by layer 6 corticothalamic neurons suppress visual cortex, Neuron 82 (2014) 474-485.

[10] J.A. Cardin, L.A. Palmer, D. Contreras, Stimulus feature selectivity in excitatory and inhibitory neurons in primary visual cortex, The Journal of neuroscience : the official journal of the Society for Neuroscience 27 (2007) 10333-10344.

[11] M.R. Cohen, A. Kohn, Measuring and interpreting neuronal correlations, Nature neuroscience 14 (2011) 811-819.

[12] J. Csicsvari, H. Hirase, A. Czurko, G. Buzsaki, Reliability and state dependence of pyramidal cell-interneuron synapses in the hippocampus: an ensemble approach in the behaving rat, Neuron 21 (1998) 179-189.

[13] Y. Dong, S. Mihalas, F. Qiu, R. von der Heydt, E. Niebur, Synchrony and the binding problem in macaque visual cortex, Journal of vision 8 (2008) 30 31-16.

[14] F. Duret, S. Shumikhina, S. Molotchnikoff, Neuron participation in a synchrony-encoding assembly, BMC neuroscience 7 (2006) 72.

[15] A. Grinvald, A. Arieli, M. Tsodyks, T. Kenet, Neuronal assemblies: single cortical neurons are obedient members of a huge orchestra, Biopolymers 68 (2003) 422-436.

[16] S.B. Hofer, H. Ko, B. Pichler, J. Vogelstein, H. Ros, H. Zeng, E. Lein, N.A. Lesica, T.D. MrsicFlogel, Differential connectivity and response dynamics of excitatory and inhibitory neurons in visual cortex, Nature neuroscience 14 (2011) 1045-1052.

[17] H. Hu, J. Gan, P. Jonas, Interneurons. Fast-spiking, parvalbumin(+) GABAergic interneurons: from cellular design to microcircuit function, Science 345 (2014) 1255263.

[18] C.P. Hung, D. Cui, Y.P. Chen, C.P. Lin, M.R. Levine, Correlated activity supports efficient cortical processing, Frontiers in computational neuroscience 8 (2014) 171.

[19] H. Ko, Neuroscience. Functional organization of synaptic connections in the neocortex, Science 346 (2014) 555.

[20] H. Ko, S.B. Hofer, B. Pichler, K.A. Buchanan, P.J. Sjostrom, T.D. Mrsic-Flogel, Functional specificity of local synaptic connections in neocortical networks, Nature 473 (2011) 8791.

[21] S.J. Kuhlman, E. Tring, J.T. Trachtenberg, Fast-spiking interneurons have an initial orientation bias that is lost with vision, Nature neuroscience 14 (2011) 1121-1123. 
[22] Y.T. Li, B.H. Liu, X.L. Chou, L.I. Zhang, H.W. Tao, Strengthening of Direction Selectivity by Broadly Tuned and Spatiotemporally Slightly Offset Inhibition in Mouse Visual Cortex, Cerebral cortex (2014).

[23] C.P. Lin, Y.P. Chen, C.P. Hung, Tuning and spontaneous spike time synchrony share a common structure in macaque inferior temporal cortex, Journal of neurophysiology 112 (2014) 856-869.

[24] D.A. McCormick, B.W. Connors, J.W. Lighthall, D.A. Prince, Comparative electrophysiology of pyramidal and sparsely spiny stellate neurons of the neocortex, Journal of neurophysiology 54 (1985) 782-806.

[25] J.E. Miller, I. Ayzenshtat, L. Carrillo-Reid, R. Yuste, Visual stimuli recruit intrinsically generated cortical ensembles, Proceedings of the National Academy of Sciences of the United States of America 111 (2014) E4053-4061.

[26] L.G. Nowak, R. Azouz, M.V. Sanchez-Vives, C.M. Gray, D.A. McCormick, Electrophysiological classes of cat primary visual cortical neurons in vivo as revealed by quantitative analyses, Journal of neurophysiology 89 (2003) 1541-1566.

[27] M. Okun, N.A. Steinmetz, L. Cossell, M.F. lacaruso, H. Ko, P. Bartho, T. Moore, S.B. Hofer, T.D. Mrsic-Flogel, M. Carandini, K.D. Harris, Diverse coupling of neurons to populations in sensory cortex, Nature 521 (2015) 511-515.

[28] B.J. Palanca, G.C. DeAngelis, Does neuronal synchrony underlie visual feature grouping?, Neuron 46 (2005) 333-346.

[29] P.R. Roelfsema, V.A. Lamme, H. Spekreijse, Synchrony and covariation of firing rates in the primary visual cortex during contour grouping, Nature neuroscience 7 (2004) 982991.

[30] E.T. Rolls, A. Treves, The neuronal encoding of information in the brain, Progress in neurobiology 95 (2011) 448-490.

[31] D.P. Schulz, M. Sahani, M. Carandini, Five key factors determining pairwise correlations in visual cortex, Journal of neurophysiology (2015) jn 0009402015.

[32] M.N. Shadlen, J.A. Movshon, Synchrony unbound: a critical evaluation of the temporal binding hypothesis, Neuron 24 (1999) 67-77, 111-125.

[33] S. Shumikhina, J. Guay, F. Duret, S. Molotchnikoff, Contextual modulation of synchronization to random dots in the cat visual cortex, Experimental brain research 158 (2004) 223-232.

[34] A. Stepanyants, J.A. Hirsch, L.M. Martinez, Z.F. Kisvarday, A.S. Ferecsko, D.B. Chklovskii, Local potential connectivity in cat primary visual cortex, Cerebral cortex 18 (2008) 13-28.

[35] M. Steriade, Neocortical cell classes are flexible entities, Nature reviews. Neuroscience 5 (2004) 121-134.

[36] H.A. Swadlow, Efferent neurons and suspected interneurons in binocular visual cortex of the awake rabbit: receptive fields and binocular properties, Journal of neurophysiology 59 (1988) 1162-1187.

[37] P. Tiesinga, T.J. Sejnowski, Cortical enlightenment: are attentional gamma oscillations driven by ING or PING?, Neuron 63 (2009) 727-732.

[38] C. van der Togt, S. Kalitzin, H. Spekreijse, V.A. Lamme, H. Super, Synchrony dynamics in monkey V1 predict success in visual detection, Cerebral cortex 16 (2006) 136-148.

[39] Y. Yoshimura, J.L. Dantzker, E.M. Callaway, Excitatory cortical neurons form fine-scale functional networks, Nature 433 (2005) 868-873. 
A
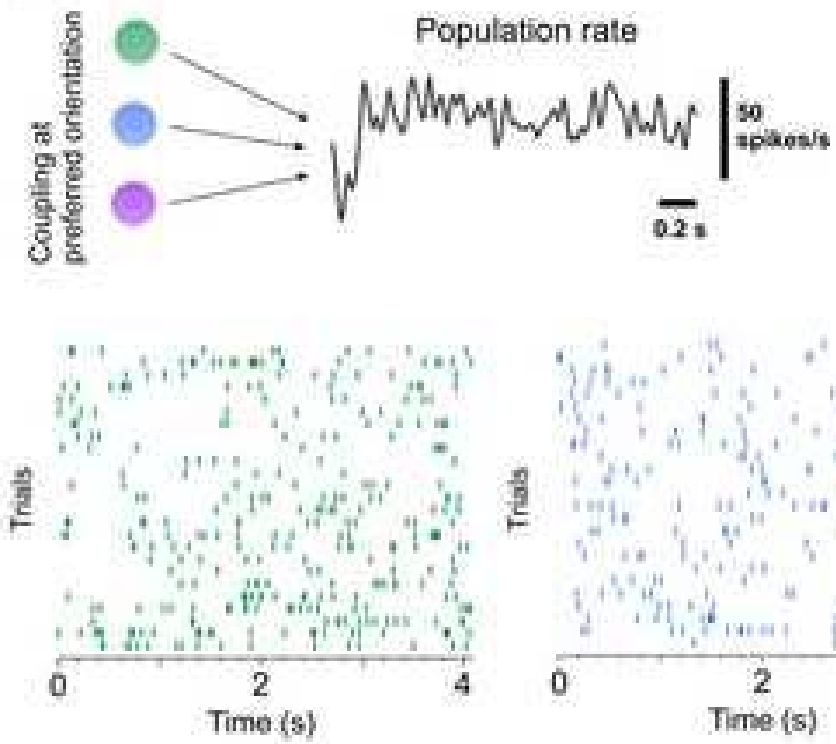
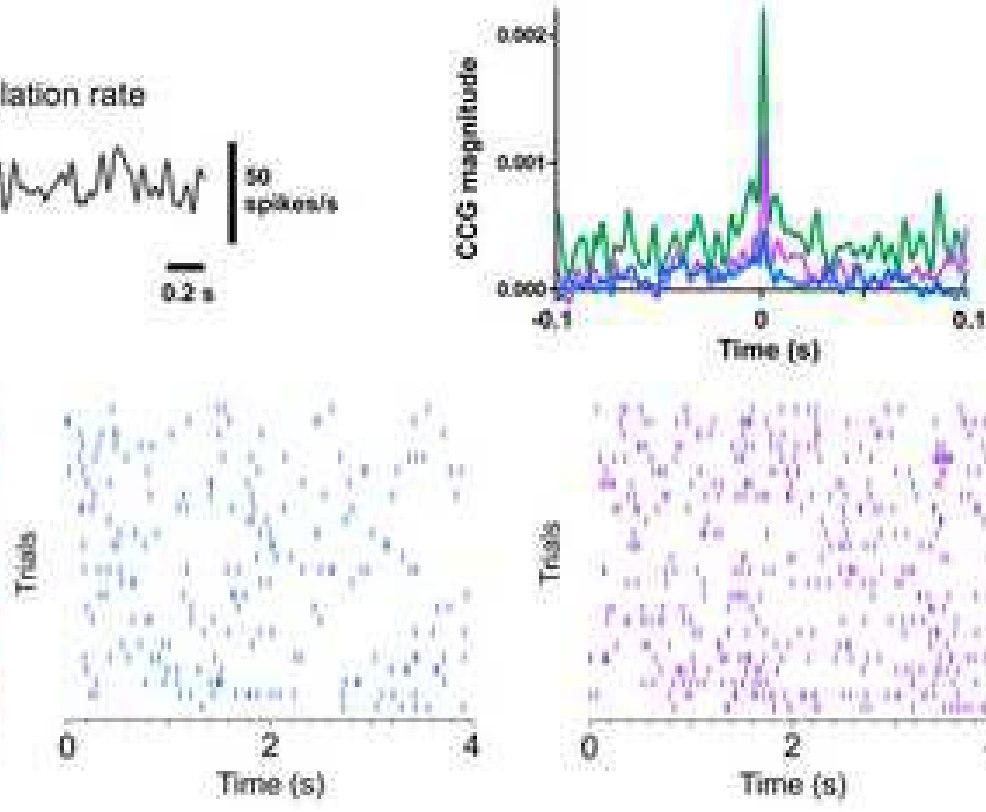

B

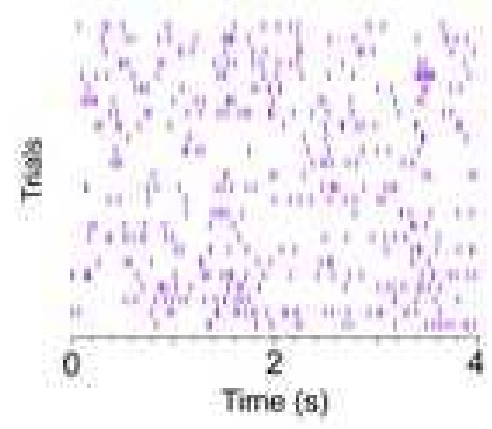

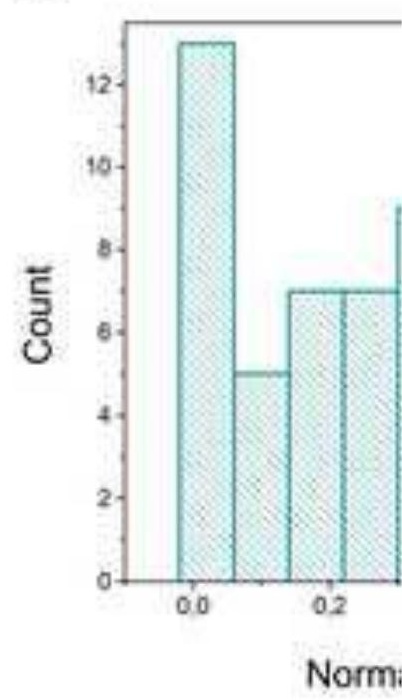


A

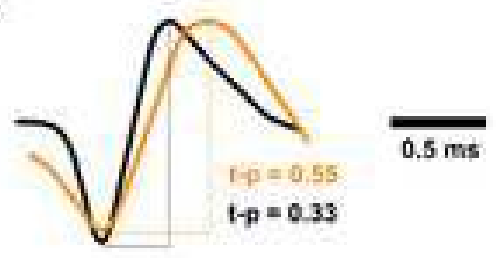

C

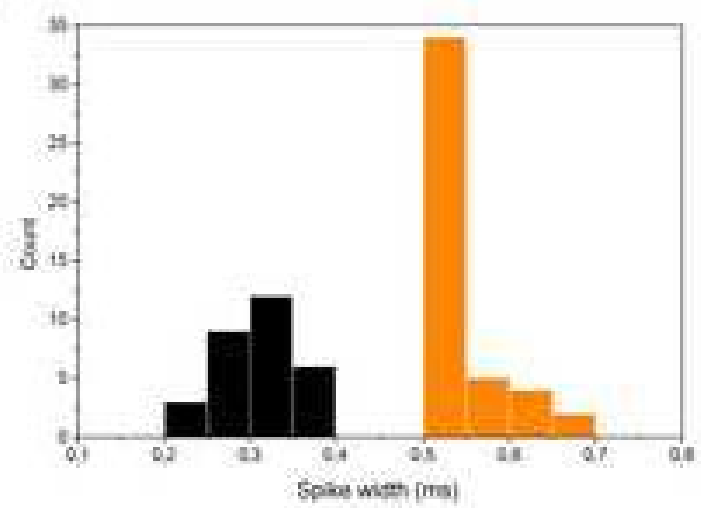

B

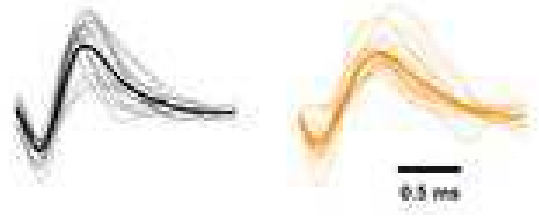

D

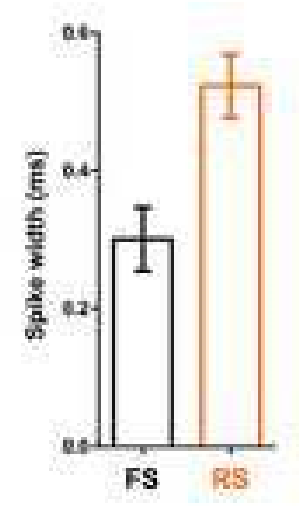

F

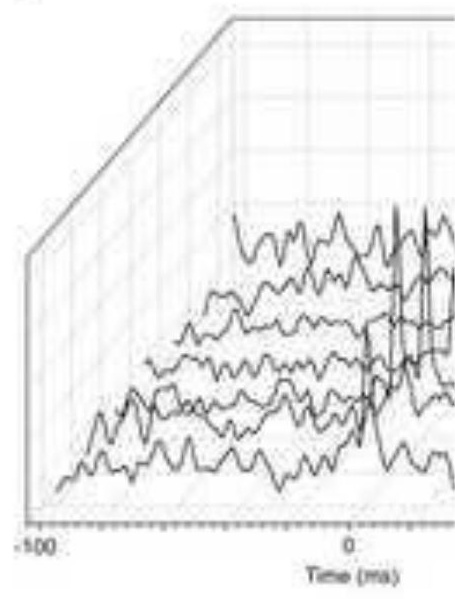

G

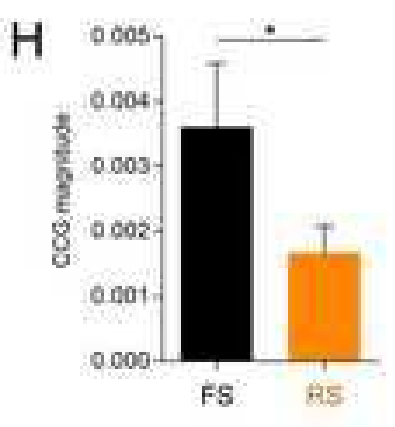

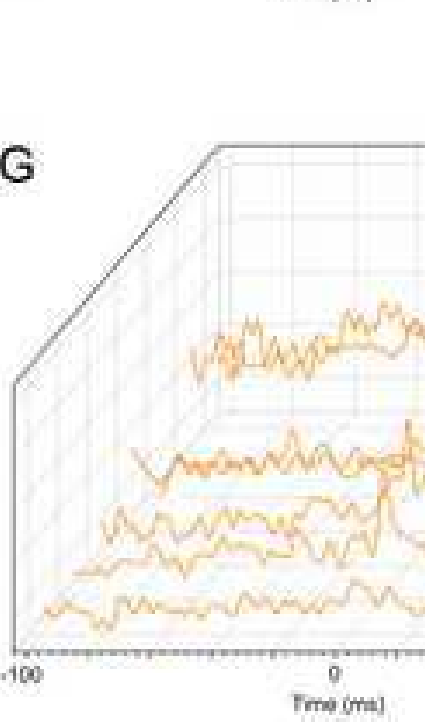
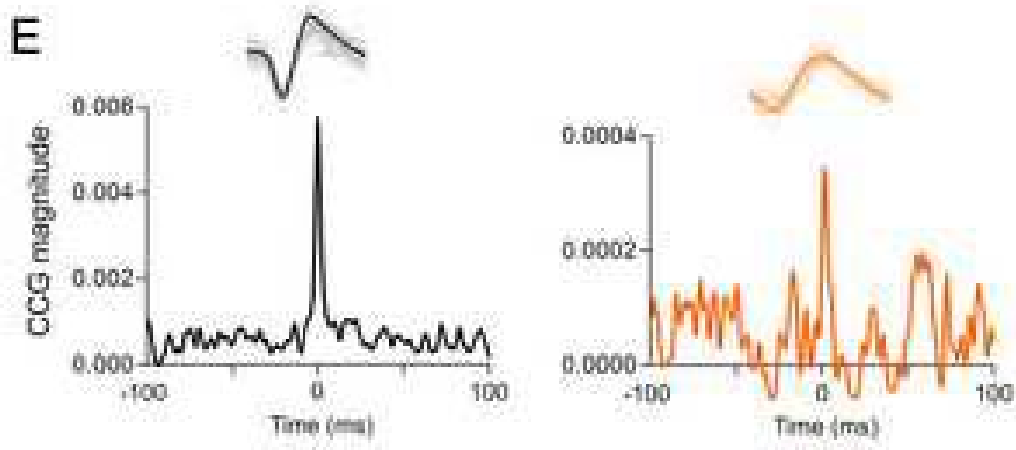
A

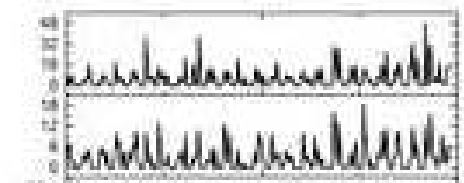

S.

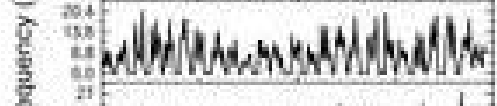

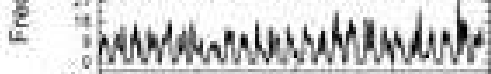

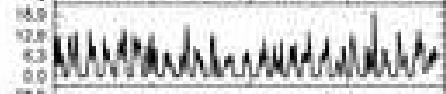

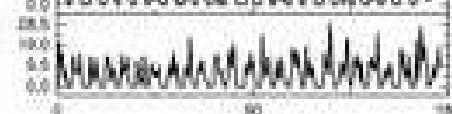
Tane (3)

D

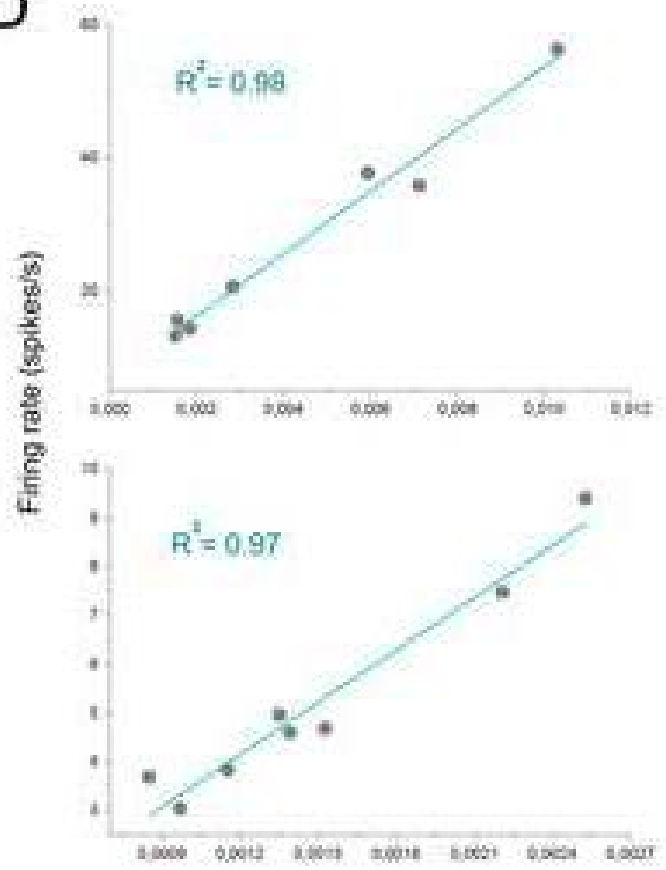

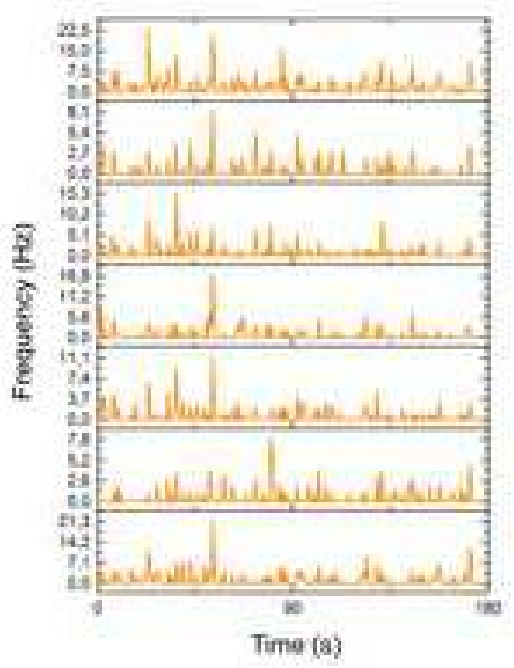

B
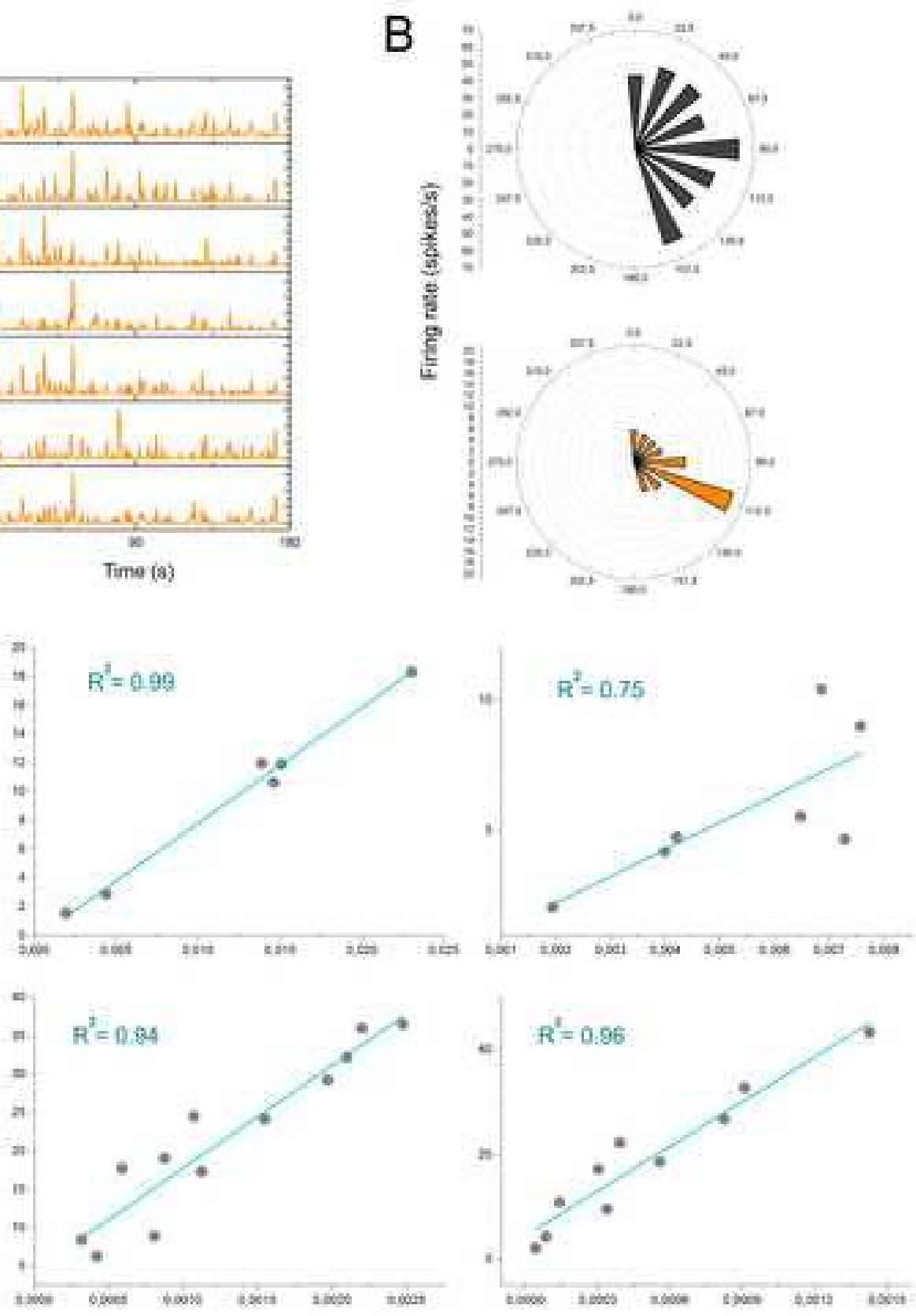

Coupling strength 


\section{Stimulus}

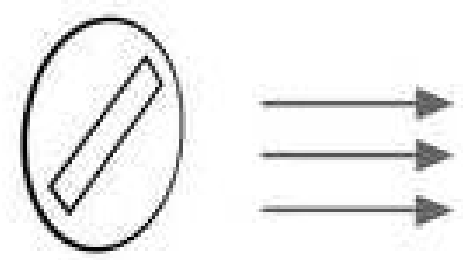

\section{Local population}

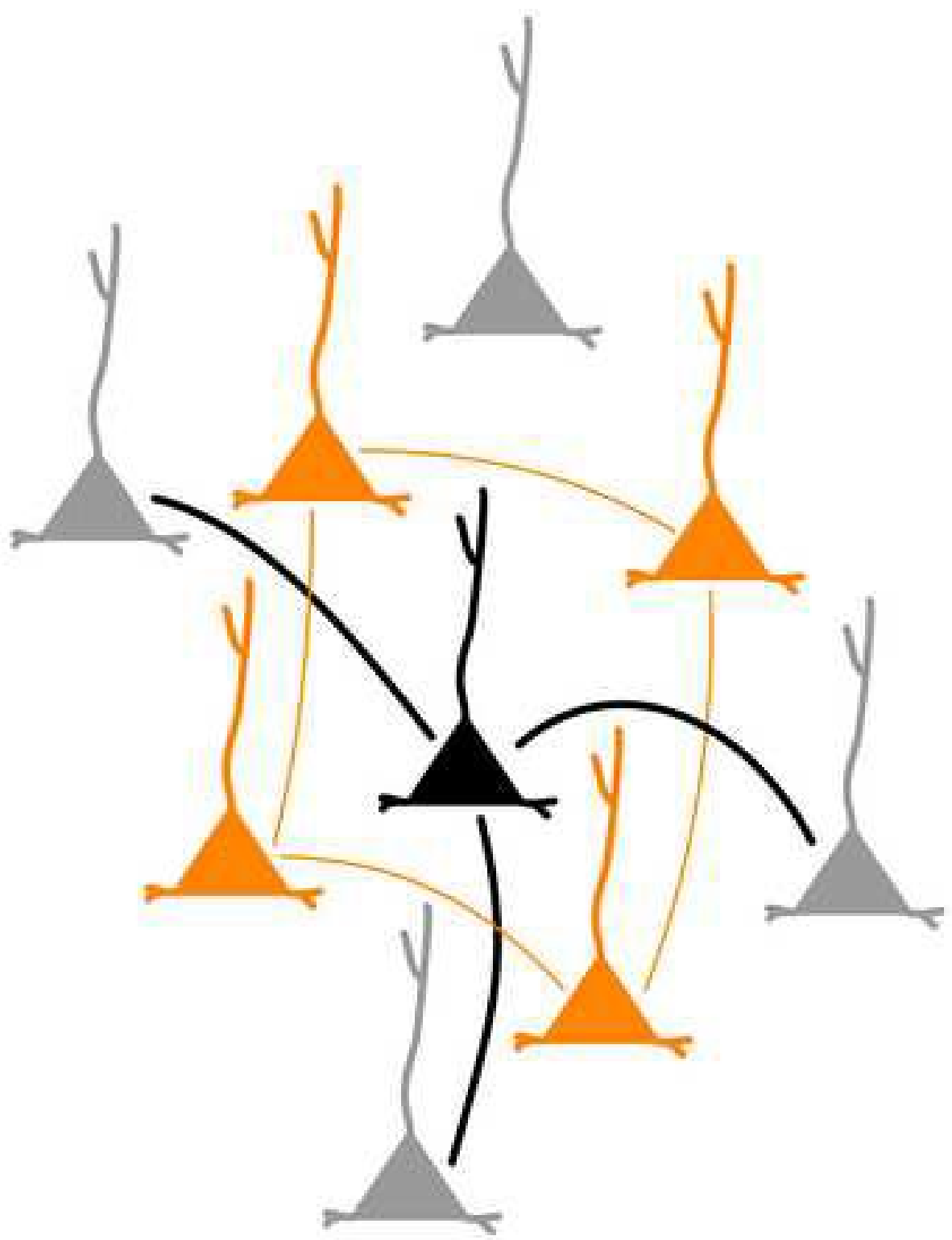

